\title{
NON-IDENTICAL DUPLICATE VIDEO DETECTION USING THE SIFT METHOD
}

\author{
Karthikeyan Vaiapury ${ }^{1}$, Pradeep K. Atrey ${ }^{1}$, Mohan S. Kankanhalli ${ }^{1}$ and Kalpathi Ramakrishnan ${ }^{2}$ \\ ${ }^{1}$ School of Computing, National University of Singapore, Republic of Singapore \\ 2 Department of Electrical Engineering, Indian Institute of Science, Bangalore
}

Keywords: NID video detection, SIFT.

\begin{abstract}
Non-Identical Duplicate video detection is a challenging research problem. Non-Identical Duplicate video are a pair of videos that are not exactly identical but are almost similar. In this paper, we evaluate two methods - Keyframe-based and Tomography-based methods to determine the Non-Identical Duplicate videos. These two methods make use of the existing scale based shift invariant (SIFT) method to find the match between the key frames in first method, and the cross-sections through the temporal axis of the videos in second method. We provide extensive experimental results and the analysis of accuracy and efficiency of the above two methods on a data set of Non-Identical Duplicate video-pair.
\end{abstract}

\section{Introduction}

Duplicate detection in multimedia content is a subject of active research within the community. The duplicate media content can exist because of two reasons - first, a copy of a video for transcoding purposes or for illegal copying of potential content; second, the consumers more often shoot multiple photos and videos of the same scene. The problem of duplicate detection in the first case is the problem of matching exactly two similar media contents, the solutions for which have been proposed using various digital signature/watermarking based methods [4], [2]. In the second case, the duplicate detection is performed by matching two media contents which are not exactly identical but almost similar (such media content are called "Non-Identical Duplicate" [5]).

The detection of Non-Identical Duplicate media content is useful for retrieval purposes such as QBE (Query by Example). For example, one may be interested in finding all the related news videos that are Non Identical Duplicate (NID) which has Abdul Kalam as the focus.

In this paper, we address the problem of detecting NonIdentical Duplicate videos. The video is a sequence of frames that have a high degree of temporal correlation among them. Each frame is an image in the two-dimensional spatial plane. The extra time dimension in video with several additional properties makes the detection of Non-Identical Duplicates in video different from that in images. We evaluate two methods for finding Non-Identical Duplicate videos - Keyframe-based method and Tomography-based method, both use the Lowe's SIFT method [8] which is highly effective in identifying and matching the interest points even for transformed images. We have performed extensive experiments and have provided the analysis of accuracy and efficiency of such approach in detecting the Non-Identical Duplicate videos.

The paper is organized as follows. We provide the related work in section 2. In section 3, we present the proposed method. Section 4 presents the extensive experimental results and analysis. Finally, we conclude the paper with a discussion on future work in section 5 .

\section{Related work}

The problem of detection of Non-Identical Duplicate media content has been studied in the past mostly in context of images $[5,3,6,7]$. The general methods of matching two video clips include the signature/watermarking based [5], [6], [2] and the distance based [4] matching methods. However, the accuracy of such method highly depends on the deployed features and moreover, they are not suitable for matching nonidentical duplicate videos due to their sensitivity to the change in color, brightness, frame format and the transformation such as scaling, rotation, down sampling etc. Significant amount of works have been done on searching near-replica, non-identical duplicates for images [8, 3, 7]. [7] used PCA SIFT based on SIFT [8] which is resistant to transformations such as scaling, rotation, down sampling etc. However the problem of finding near replica using scale invariant method has been addressed only for the images.

In this work, we have addressed the Non-Identical Duplicate problem for videos using the keyframe-based and the tomography-based SIFT methods. The SIFT method [8] is used for finding similarity match between any two frames and it provides significantly accurate results, though it is computational expensive. This method is used because of its robustness to variation in scale, rotation, affine distortion and $3 \mathrm{D}$ viewpoint etc. The steps used in generating the features of video key frames include a) Finding the scale and orientation invariant interest points using DoG (Difference of Gaussian function), b) Selecting the key points based on stability, c) Assigning the orientation, and d) Key point descriptor. 


\section{Proposed work}

We evaluate two methods - Keyframe-based and Tomographybased methods to find whether or not the given videos are Non-Identical Duplicates. Both the methods use SIFT-method for matching two (key-frames) in Keyframe-based method and (cross-sections) in Tomography-based method.

\subsection{Keyframe-based method}

In order to determine whether or not the given two videos are Non-Identical Duplicate, the Keyframe-based method works as follows -

1. Given the two input videos (say V1 and V2), we extract the key frames from both the videos using the simple differencing and thresholding method similar to what is used in [2]. Let $\mathrm{KF} 1=\left\{k_{1 i}\right\}, 1 \leq i \leq n_{1}$ and $\mathrm{KF} 2=$ $\left\{k_{2 j}\right\}, 1 \leq j \leq n_{2}$ be the sets of key frames of videos $\mathrm{V} 1$ and $\mathrm{V} 2$, respectively; where $n_{1}$ and $n_{2}$ are the total number of key frames in videos V1 and V2, respectively.

2. The similarity matches between all the pairs $\left(k_{1 i}, k_{2 j}\right)$, $1 \leq i \leq n_{1}, 1 \leq j \leq n_{2}$ are obtained using the SIFT method [8]. As a result of this, we obtain a matrix $M_{i j}$, $1 \leq i \leq n_{1}, 1 \leq j \leq n_{2}$. The match score $M_{i j}$ is computed using the following equation -

$$
M_{i j}=2 \times \frac{m_{i j}}{P_{i}+P_{j}}
$$

where, $m_{i j}$ is the number of match points between the frames $i$ and $j$, and $P_{i}$ and $P_{j}$ are the number of key points found using SIFT method for the frame $i$ and frame $j$, respectively.

3. Finally, we obtain the Overall Match Score $(O M S)$ between the two videos by averaging all the match scores $M_{i j}$ in a diagonal band of a given width. Precisely, the $O M S_{k}$ for a band of width $k$ is computed as -

$$
O M S_{k}=\operatorname{mean}\left(M_{i j}\right)
$$

where, $1 \leq i \leq n_{1}, 1 \leq j \leq n_{2}$ and $i=j-(k-1) / 2$.

In case, $n_{1} \neq n_{2}$, we repeat the diagonal band until the last element of $n_{1}$ or $n_{2}$ whichever is higher, and compute the Overall Match Score by averaging the match scores of all the bands.

\subsection{Tomography-based method}

We also explored the use of tomography-based [1] matching method to find the Non-Identical Duplicate videos.

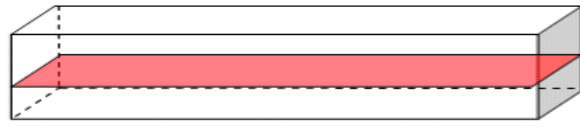

(a)

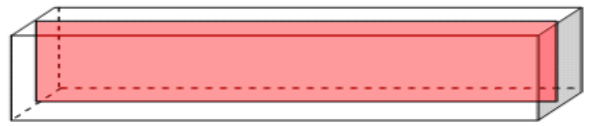

(b)

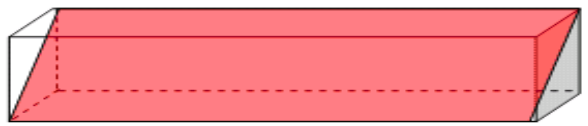

(c)

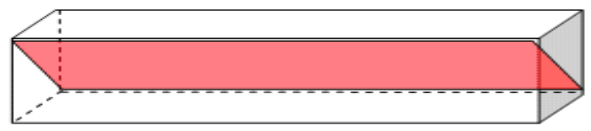

Figure 1: Four cross-sections used in our experiments (a) Middle horizontal (b) Middle vertical (c) Diagonal 1 and (d) Diagonal 2

Tomography refers to finding the cross-sections through the temporal axis of the video. In this method, instead of using key frames, we used the four (middle-horizontal, middle-vertical, and the two diagonal) cross-sections through the temporal axis of the two videos as shown in figure 1, and then compared them with the similar cross-sections of the other video using SIFT-method. The Overall Match Score (OMS) is computed by averaging the individual match scores of all the four cross-sections matching.

\section{Experimental Results}

The extensive experiments are performed to analyze the accuracy and efficiency of SIFT method in detecting the Non-Identical Duplicate videos. For our experiments, we used Pentium-IV $2.4 \mathrm{GHz}$ with $512 \mathrm{MB}$ RAM. We prepared a data-set of 16 Non-Identical Duplicate video-pair (shown in figure 2). All the videos were shot using a Fujifilm digital camera (Model A210) with $320 \times 240$ resolution. Note that since no standard data set on NID-video pair is publicly available, we have used our own data set. The past works have used the "Run-Lola-Run" data set which provides only NID-images not the videos. We also report results on the "Run-Lola-Run" data set considering the NID-images as a representative to the video.

\subsection{Experiments on our data set}

In keyframe-based SIFT method, we first extract the key frames from each video. The total number of frames in each video and the number of key frames extracted from them are shown 

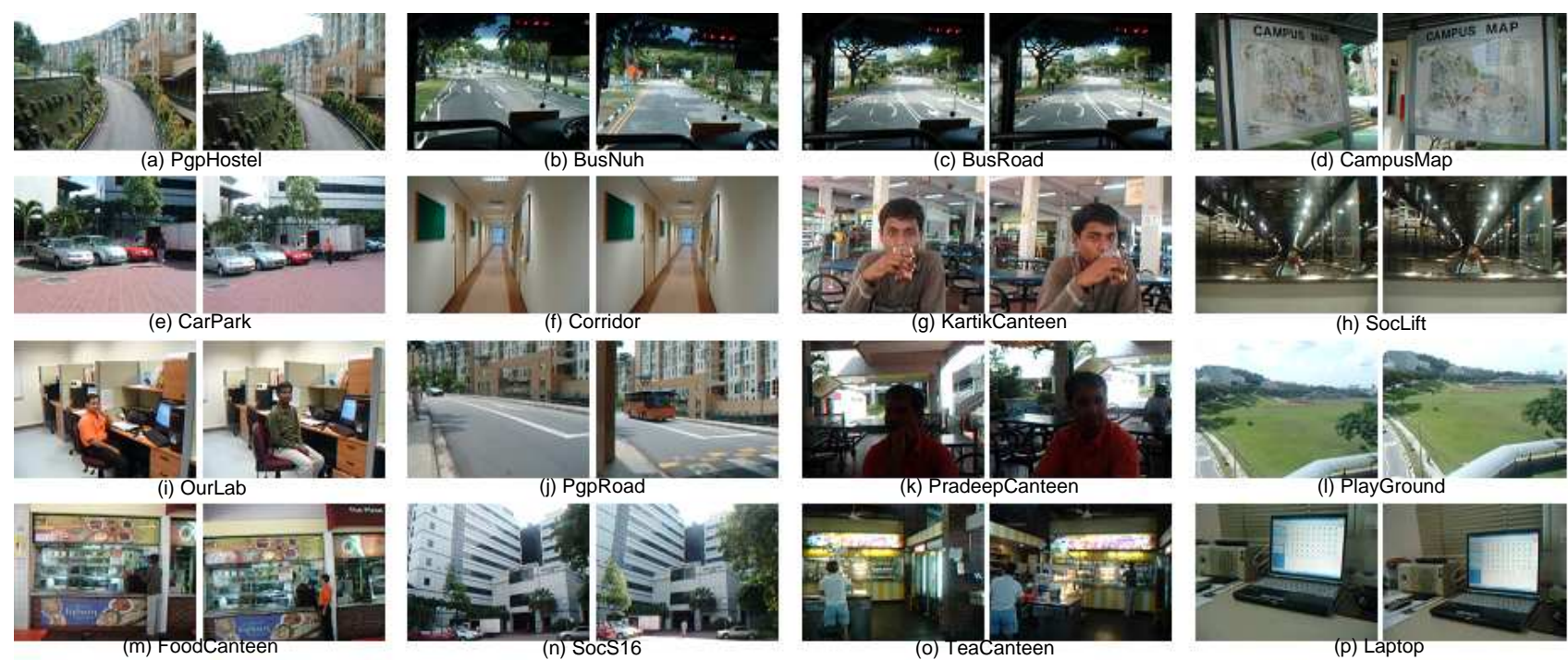

Figure 2: The key frames of 16 Non-Identical Duplicate video pair of our data set

Table 1: Details of Non-Identical Duplicate videos used in our experiment

\begin{tabular}{lcccc}
\hline NID Video pair & $\begin{array}{c}\text { Total number } \\
\text { of frames }\end{array}$ & $\begin{array}{c}\text { Duration } \\
\text { of clips } \\
\text { (in seconds) }\end{array}$ & $\begin{array}{c}\text { Number of } \\
\text { key frames } \\
\text { (Set 1) } \\
n_{1}, n_{2}\end{array}$ & $\begin{array}{c}\text { Number of } \\
\text { key frames } \\
\text { (Set 2) } \\
n_{1}, n_{2}\end{array}$ \\
\hline PgpHostel & 99,89 & 9,8 & 23,16 & 6,4 \\
BusNuh & 40,40 & 3,3 & 10,10 & 1,2 \\
BusRoad & 50,70 & 4,6 & 14,22 & 5,8 \\
CampusMap & 120,100 & 12,9 & 12,5 & 2,1 \\
CarPark & 70,90 & 6,8 & 13,16 & 5,6 \\
Corridor & 60,70 & 5,6 & 3,3 & 1,1 \\
KartikCanteen & 60,60 & 5,5 & 13,15 & 4,5 \\
SocLift & 30,30 & 3,3 & 3,1 & 1,1 \\
OurLab & 80,100 & 7,9 & 21,14 & 12,8 \\
PgpRoad & 70,30 & 6,3 & 10,17 & 3,7 \\
PradeepCanteen & 120,130 & 14,16 & 43,69 & 20,30 \\
PlayGround & 90,80 & 8,7 & 5,3 & 3,1 \\
FoodCanteen & 110,130 & 10,12 & 30,37 & 10,11 \\
SocS16 & 100,90 & 9,8 & 24,28 & 11,12 \\
TeaCanteen & 60,40 & 5,4 & 7,4 & 2,1 \\
Laptop & 40,60 & 4,5 & 3,4 & 1,2 \\
\hline
\end{tabular}

in Table 1. We have obtained two sets (Set 1 and Set 2) of key frames using different threshold values. This is done with the aim of analyzing how much computational efforts can be saved while maintaining the accuracy. We compared each video-pair using SIFT method and computed the Overall Match Score (OMS) using equation (2). The value of width $k$ of band is chosen as 1 and 3. In other words, $k=1$ allows to compare each keyframe in one video with the corresponding key frame in the other video; while with $k=3$, we compare each key frame in one video with the one previous and one next keyframe in addition to the corresponding key frame of the other video.

We used a Bayesian classifier to categorize a given input videopair into one of the two classes - NID pair and Non-NID pair.
The Bayesian classifier is trained based on Overall Match Score (OMS) obtained for a set of NID-pair and Non-NID pair.

We have performed experiment using the Keyframe-based SIFT method on all the video pairs (shown in figure 2) of original resolution i.e. $320 \times 240$. The same experiment is performed on the video pairs by down-grading them to lower resolutions i.e. $160 \times 120,80 \times 60,40 \times 30$ and $20 \times 15$. We did this experiment to observe how the accuracy and efficiency are affected by matching low resolution video pairs. The Overall Match Score is computed for the two different values of width $k$ of the diagonal band (refer to section 3.1), i.e. for $k=1$ and $k=3$. The results for $\mathrm{OMS}_{1}$ (for $k=1$ ) and for $\mathrm{OMS}_{3}$ (for $k=3$ ) are reported in Table 2 and Table 3, respectively. In Table 2 and Table 3, each entry for a particular resolution of the video pair has three values - Overall Match Score $\left(\mathrm{OMS}_{k}\right)$ (multiplied by $10^{4}$ ), the time $\left(T_{k}\right)$ taken in computing this score and the probability $\left(P_{k}\right)$ of this pair belonging to a NID-pair class, with $k$ being the width of diagonal band.

We also performed experiment on the video pairs of original resolution (i.e. $320 \times 240$ ) by using the Set 2 of key frames (as described in Table 1). The results for this experiment are reported in Table 4.

The experiments are also performed for Tomography method (as described in section 3.2). The results are shown in Table 5.

The overall observations from the obtained results are-

1. From our experiments, we clearly found that SIFT based method is effective and works well for detecting NonIdentical Duplicate videos. 
Table 2: Overall Match Score (OMS1 with $k=1$ ) for NID video pairs (with Set 1 of key frames) at varying resolutions using Keyframe-based SIFT method

\begin{tabular}{|c|c|c|c|c|c|c|c|c|c|c|c|c|c|c|c|}
\hline \multirow{2}{*}{$\begin{array}{c}\text { NID } \\
\text { Video pair }\end{array}$} & \multicolumn{3}{|c|}{$320 \times 240$} & \multicolumn{3}{|c|}{$160 \times 120$} & \multicolumn{3}{|c|}{$80 \times 60$} & \multicolumn{3}{|c|}{$40 \times 30$} & \multicolumn{3}{|c|}{$20 \times 15$} \\
\hline & $\mathrm{OMS}_{1}$ & $\begin{array}{c}T_{1} \\
(\mathrm{Sec})\end{array}$ & $P_{1}$ & $\mathrm{OMS}_{1}$ & $\begin{array}{c}T_{1} \\
(\mathrm{Sec})\end{array}$ & $P_{1}$ & $\mathrm{OMS}_{1}$ & $\begin{array}{c}T_{1} \\
(\mathrm{Sec})\end{array}$ & $P_{1}$ & $\mathrm{OMS}_{1}$ & $\begin{array}{c}T_{1} \\
(\mathrm{Sec})\end{array}$ & $P_{1}$ & $\mathrm{OMS}_{1}$ & $\begin{array}{c}T_{1} \\
(\mathrm{Sec})\end{array}$ & $P_{1}$ \\
\hline PgpHostel & 508 & 158 & 0.9998 & 823 & 136 & 0.9999 & 743 & 122 & 1.0000 & 693 & 109 & 0.9999 & 517 & 33 & 0.9995 \\
\hline BusNuh & 55 & 42 & 0.9423 & 98 & 33 & 0.9970 & 109 & 27 & 0.9847 & 358 & 25 & 0.9993 & 0 & 2 & 0.0014 \\
\hline BusRoad & 31 & 248 & 0.7625 & 81 & 80 & 0.9637 & 66 & 72 & 0.9482 & 143 & 66 & 0.9929 & 0 & 1 & 0.0014 \\
\hline CampusMap & 227 & 50 & 0.9980 & 220 & 43 & 0.9966 & 131 & 18 & 0.9902 & 0 & 12 & 0.0026 & 0 & 2 & 0.0014 \\
\hline CarPark & 408 & 108 & 0.9996 & 789 & 55 & 0.9999 & 964 & 44 & 1.0000 & 842 & 25 & 0.9999 & 220 & 23 & 0.9971 \\
\hline Corridor & 3744 & 4 & 1.0000 & 4822 & 4 & 1.0000 & 6631 & 2 & 1.0000 & 6274 & 2 & 1.0000 & 4111 & 2 & 1.0000 \\
\hline KartikCanteen & 706 & 42 & 0.9999 & 800 & 20 & 0.9999 & 478 & 16 & 0.9996 & 296 & 11 & 0.9988 & 0 & 1 & 0.0014 \\
\hline SocLift & 6266 & 3 & 1.0000 & 6950 & 3 & 1.0000 & 1268 & 3 & 1.0000 & 1182 & 3 & 1.0000 & 222 & 3 & 0.9971 \\
\hline OurLab & 369 & 139 & 0.9994 & 125 & 36 & 0.9858 & 0 & 16 & 0.0027 & 0 & 10 & 0.0026 & 0 & 1 & 0.0014 \\
\hline PgpRoad & 559 & 168 & 0.9998 & 491 & 45 & 0.9996 & 382 & 36 & 0.9993 & 167 & 30 & 0.9951 & 0 & 1 & 0.0014 \\
\hline PradeepCanteen & 61 & 2536 & 0.9551 & 92 & 2408 & 0.9733 & 35 & 727 & 0.7409 & 0 & 614 & 0.0026 & 0 & 2 & 0.0014 \\
\hline PlayGround & 3978 & 12 & 1.0000 & 5140 & 10 & 1.0000 & 5886 & 9 & 1.0000 & 6477 & 6 & 1.0000 & 0 & 1 & 0.0014 \\
\hline FoodCanteen & 2503 & 366 & 1.0000 & 1383 & 343 & 1.0000 & 1831 & 326 & 1.0000 & 777 & 221 & 1.0000 & 0 & 2 & 0.0014 \\
\hline SocS16 & 3235 & 158 & 1.0000 & 2981 & 152 & 1.0000 & 2978 & 148 & 1.0000 & 3007 & 141 & 1.0000 & 0 & 1 & 0.0014 \\
\hline TeaCanteen & 890 & 17 & 1.0000 & 584 & 14 & 0.9997 & 648 & 10 & 0.9999 & 1052 & 9 & 1.0000 & 500 & 6 & 0.9995 \\
\hline Laptop & 2763 & 6 & 1.0000 & 4668 & 5 & 1.0000 & 4283 & 4 & 1.0000 & 5576 & 3 & 1.0000 & 746 & 2 & 1.0000 \\
\hline
\end{tabular}

2. Keyframe-based method performs with a decent accuracy and is much faster than the simple approach of comparing two videos by matching each frame of one video with the each frame of the other video within a certain width of band. We verified it by comparing the NonIdentical Duplicate pair of the video 'PgpHostel'. The computation time for matching (89 frames of PgpHostel1 and 99 frames of PgpHostel2) is found around 270 minutes, which is significantly greater than the average computation time recorded for keyframe-based method.

3. We have observed (from Table 2 and Table 3) that the computation time significantly decreases for the lower resolution video pairs (as also can be seen in figure 3), and it also provides reasonably accurate results upto a certain resolution. This is because, by reducing the resolution of the frame, the number of key points that are invariant are also reduced but lesser number of match points are reduced. As can be seen from figure 4, for $\mathrm{OMS}_{1}$, the accuracy remains more than $83 \%$ upto resolution $40 \times 30$ and it degrades to $37 \%$ with resolution $20 \times 15$. This indicates that SIFT-based method works even for matching the low-resolution video pairs.

4. It is also observed that the overall accuracy (for our data set) is found to be better with $k=3$ compared to that with $k=1$. As can be seen in figure 4 , for video pairs of resolution $40 \times 30$, the accuracy for $\mathrm{OMS}_{3}$ is 93.75\% which is higher compared to the accuracy for $\mathrm{OMS}_{1}$ which is $81.25 \%$.

5. It is also interesting to note that, for Set 2 of key frames, the SIFT method performed well i.e. with accuracies of $87.5 \%$ (for $\mathrm{OMS}_{1}$ ) and $100 \%$ (for $\mathrm{OMS}_{3}$ ) (Refer to Table 4). Also, it provides a significant amount of gain in the computation time (i.e. around 10 times faster).

6. After comparing the Tomography-based method with the Keyframe-based method, we found that Tomographybased method does not perform well for the data set

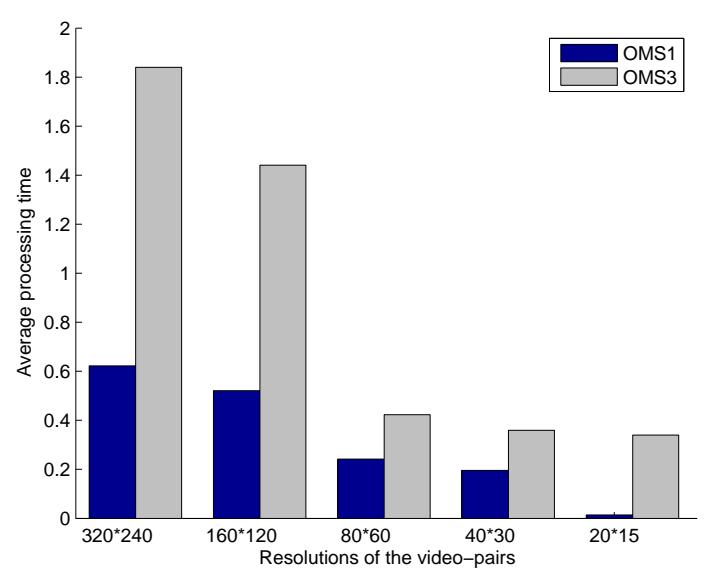

Figure 3: Average processing time (in seconds) for matching a single frame-pair of the videos of different resolutions

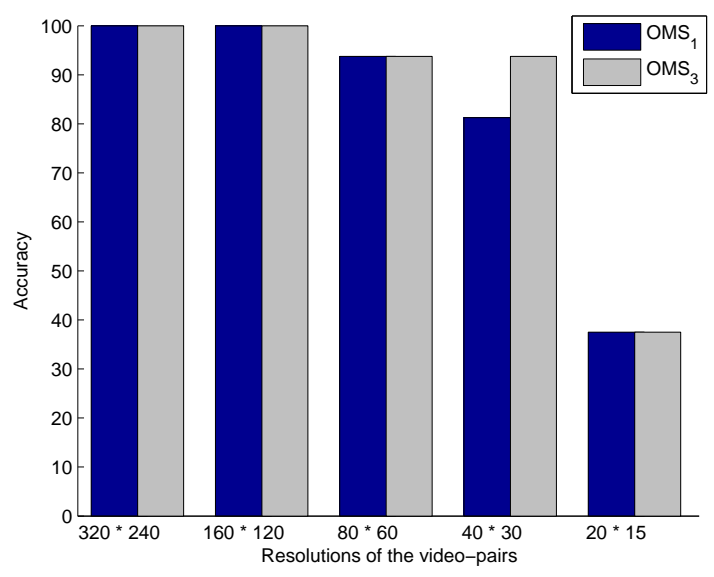

Figure 4: Accuracy of NID video pair detection vs. Resolution of video pairs 
Table 3: Overall Match Score (OMS3 with $k=3$ ) for NID video pairs (with Set 1 of key frames) at varying resolutions using Keyframe-based SIFT method

\begin{tabular}{|c|c|c|c|c|c|c|c|c|c|c|c|c|c|c|c|}
\hline \multirow{2}{*}{$\begin{array}{c}\text { NID } \\
\text { Video pair }\end{array}$} & \multicolumn{3}{|c|}{$320 \times 240$} & \multicolumn{3}{|c|}{$160 \times 120$} & \multicolumn{3}{|c|}{$80 \times 60$} & \multicolumn{3}{|c|}{$40 \times 30$} & \multicolumn{3}{|c|}{$20 \times 15$} \\
\hline & $\mathrm{OMS}_{1}$ & $\begin{array}{c}T_{1} \\
(\mathrm{Sec})\end{array}$ & $P_{1}$ & $\mathrm{OMS}_{1}$ & $\begin{array}{c}T_{1} \\
(\mathrm{Sec})\end{array}$ & $P_{1}$ & $\mathrm{OMS}_{1}$ & $\begin{array}{c}T_{1} \\
(\mathrm{Sec})\end{array}$ & $P_{1}$ & $\mathrm{OMS}_{1}$ & $\begin{array}{c}T_{1} \\
(\mathrm{Sec})\end{array}$ & $P_{1}$ & $\mathrm{OMS}_{1}$ & $\begin{array}{c}T_{1} \\
(\mathrm{Sec})\end{array}$ & $P_{1}$ \\
\hline PgpHostel & 485 & 470 & 0.9993 & 787 & 402 & 0.9996 & 826 & 263 & 1.0000 & 611 & 243 & 0.9998 & 458 & 107 & 0.9998 \\
\hline BusNuh & 63 & 110 & 0.8848 & 125 & 80 & 0.9858 & 180 & 72 & 0.9949 & 406 & 67 & 0.9994 & 0 & 2 & 0.0014 \\
\hline BusRoad & 36 & 740 & 0.5937 & 66 & 234 & 0.9337 & 68 & 102 & 0.9472 & 221 & 99 & 0.9971 & 0 & 1 & 0.0014 \\
\hline CampusMap & 252 & 148 & 0.9960 & 278 & 105 & 0.9980 & 171 & 27 & 0.9942 & 94 & 24 & 0.9776 & 0 & 2 & 0.0014 \\
\hline CarPark & 502 & 320 & 0.9994 & 900 & 110 & 1.0000 & 916 & 65 & 1.0000 & 960 & 35 & 1.0000 & 658 & 28 & 1.0000 \\
\hline Corridor & 3490 & 11 & 1.0000 & 4581 & 6 & 1.0000 & 5804 & 3 & 1.0000 & 6142 & 2 & 1.0000 & 692 & 2 & 1.0000 \\
\hline KartikCanteen & 1179 & 124 & 1.0000 & 1403 & 55 & 1.0000 & 920 & 36 & 1.0000 & 833 & 23 & 0.9999 & 0 & 1 & 0.0014 \\
\hline SocLift & 786 & 9 & 0.9999 & 1647 & 3 & 1.0000 & 1612 & 3 & 1.0000 & 1479 & 3 & 1.0000 & 1479 & 3 & 1.0000 \\
\hline OurLab & 367 & 395 & 0.9985 & 131 & 112 & 0.9902 & 0 & 36 & 0.0027 & 0 & 29 & 0.0026 & 0 & 1 & 0.0014 \\
\hline PgpRoad & 718 & 502 & 0.9998 & 669 & 127 & 0.9998 & 371 & 56 & 0.9992 & 240 & 47 & 0.9976 & 0 & 1 & 0.0014 \\
\hline PradeepCanteen & 512 & 7520 & 0.9994 & 77 & 6875 & 0.9545 & 43 & 1263 & 0.8335 & 60 & 1120 & 0.9327 & 0 & 2 & 0.001 \\
\hline PlayGround & 3888 & 24 & 1.0000 & 5009 & 22 & 1.0000 & 5696 & 19 & 1.0000 & 5909 & 12 & 1.0000 & 0 & 1 & 0.0014 \\
\hline FoodCanteen & 2490 & 1082 & 1.0000 & 1359 & 772 & 1.0000 & 1766 & 451 & 1.0000 & 717 & 359 & 0.9999 & 0 & 2 & 0.0014 \\
\hline SocS16 & 3852 & 470 & 1.0000 & 3075 & 433 & 1.0000 & 3032 & 306 & 1.0000 & 2745 & 230 & 1.0000 & 0 & 1 & 0.0014 \\
\hline TeaCanteen & 402 & 50 & 0.9988 & 507 & 48 & 0.9996 & 473 & 45 & 0.9996 & 1381 & 41 & 1.0000 & 1031 & 41 & 1.0000 \\
\hline Laptop & 3920 & 17 & 1.0000 & 4905 & 11 & 1.0000 & 5686 & 9 & 1.0000 & 6153 & 6 & 1.0000 & 769 & 6 & 1.0000 \\
\hline
\end{tabular}

Table 4: Overall Match Score (OMS) for different pair of videos (for Set 2 of key frames) at resolution $320 \times 240$ using Keyframe-based SIFT method

\begin{tabular}{lcccccc}
\hline NID Video pair & $\mathrm{OMS}_{1}$ & $\begin{array}{c}T_{1} \\
(\mathrm{Sec})\end{array}$ & $P_{1}$ & $\mathrm{OMS}_{3}$ & $\begin{array}{c}T_{3} \\
(\mathrm{Sec})\end{array}$ & $P_{3}$ \\
\hline PgpHostel & 467 & 11 & 0.9997 & 484 & 33 & 0.9997 \\
BusNuh & 23 & 2 & 0.1764 & 63 & 6 & 0.9201 \\
BusRoad & 33 & 21 & 0.4973 & 36 & 63 & 0.6858 \\
CampusMap & 160 & 2 & 0.9887 & 348 & 6 & 0.9991 \\
CarPark & 437 & 7 & 0.9996 & 324 & 21 & 0.9989 \\
Corridor & 486 & 2 & 0.9998 & 486 & 2 & 0.9997 \\
KartikCanteen & 726 & 6 & 1.0000 & 714 & 17 & 1.0000 \\
SocLift & 2059 & 2 & 1.0000 & 2059 & 2 & 1.0000 \\
OurLab & 401 & 45 & 0.9994 & 390 & 135 & 0.9994 \\
PgpRoad & 847 & 16 & 1.0000 & 592 & 48 & 0.9999 \\
PradeepCanteen & 56 & 280 & 0.8382 & 467 & 835 & 0.9997 \\
PlayGround & 1137 & 3 & 1.0000 & 3888 & 9 & 1.0000 \\
FoodCanteen & 1323 & 14 & 1.0000 & 1388 & 40 & 1.0000 \\
SocS16 & 1622 & 15 & 1.0000 & 1686 & 45 & 1.0000 \\
TeaCanteen & 684 & 2 & 1.0000 & 356 & 6 & 0.9992 \\
Laptop & 1411 & 2 & 1.0000 & 2079 & 6 & 1.0000 \\
\hline \multicolumn{7}{c}{ OMS $_{1}:$ Accuracy $=87.5 \%$, OMS $_{3}:$ Accuracy $=100 \%$} \\
\end{tabular}

provided in figure 2. Using Tomography-based method, the average OMS for NID video-pair and Non-NID videopair was found 9.6 and 5.8, respectively, which was not good enough to distinguish between NID and Non-NID video-pair (Refer to Table 5). This might be because these videos are shot by keeping the camera in hand, which constantly changes the view point. In such videos, finding the correctly matching cross-section in two videos is difficult.

7. We also computed the Overall Match Score for NonNID videos using SIFT-based method. The results are provided in Table 6 . The results clearly show that SIFT-based method can detect Non-NID video pair with a significantly high accuracy (82\% and $91 \%$, with bandwidth $k=1$ and $k=3$, respectively).
Table 5: Overall Match Score (OMS) for different pair of videos (for Set 1 of key frames) at resolution $320 \times 240$ using Tomography-based SIFT method

\begin{tabular}{lcc}
\hline NID Video pair & OMS $_{1}$ & $T_{1}(\mathrm{Sec})$ \\
\hline PgpHostel & 15 & 40 \\
BusNuh & 10 & 30 \\
BusRoad & 9 & 32 \\
CampusMap & 6 & 45 \\
CarPark & 14 & 36 \\
KartikCanteen & 8 & 34 \\
SocLift & 5 & 32 \\
OurLab & 12 & 40 \\
PgpRoad & 9 & 34 \\
PradeepCanteen & 10 & 47 \\
PlayGround & 11 & 36 \\
FoodCanteen & 5 & 32 \\
SocS16 & 8 & 30 \\
TeaCanteen & 7 & 30 \\
Laptop & 15 & 32 \\
\hline
\end{tabular}

Table 6: Overall Match Score (OMS) for Non-NID pair of videos (for Set 1 of key frames) at resolution $320 \times 240$ using Keyframe-based SIFT method

\begin{tabular}{|c|c|c|c|c|c|c|c|}
\hline Video $1\left(n_{1}\right)$ & Video $2\left(n_{1}\right)$ & $\begin{array}{c}\mathrm{OMS}_{1} \\
(\mathrm{Sec})\end{array}$ & $T_{1}$ & $P_{1}$ & $\begin{array}{c}\mathrm{OMS}_{3} \\
(\mathrm{Sec})\end{array}$ & $T_{3}$ & $P_{3}$ \\
\hline BusNuh (10) & BusRoad (14) & 43 & 70 & 0.8943 & 69 & 201 & 0.9072 \\
\hline BusRoad (14) & CampusMap (12) & 8 & 82 & 0.0839 & 8 & 240 & 0.1083 \\
\hline CampusMap (12) & CarPark (13) & 8 & 68 & 0.0839 & 8 & 194 & 0.1083 \\
\hline CarPark (13) & Corridor (3) & 9 & 19 & 0.0433 & 10 & 55 & 0.0542 \\
\hline Corridor (3) & KartikCanteen (13) & 0 & 19 & 0.0039 & 3 & 55 & 0.2778 \\
\hline OurLab (21) & SoCLift (3) & 14 & 20 & 0.0536 & 11 & 58 & 0.0330 \\
\hline SoCLift (3) & PlayGround (5) & 0 & 7 & 0.0039 & 5 & 7 & 0.2076 \\
\hline PlayGround (5) & PradeepCanteen (43) & 4 & 110 & 0.3039 & 4 & 305 & 0.2427 \\
\hline PradeepCanteen (43) & FoodCanteen (30) & 8 & 612 & 0.0839 & 9 & 1804 & 0.0796 \\
\hline FoodCanteen (30) & SoCS16 (24) & 5 & 340 & 0.2461 & 3 & 1270 & 0.2778 \\
\hline SoCS16 (24) & TeaCanteen (7) & 26 & 84 & 0.6393 & 28 & 245 & 0.3646 \\
\hline
\end{tabular}



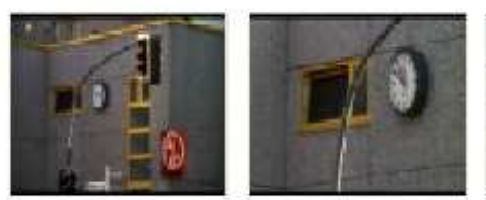

Clock1

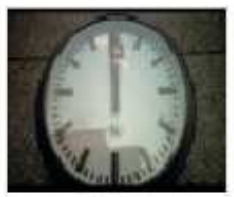

Clock4

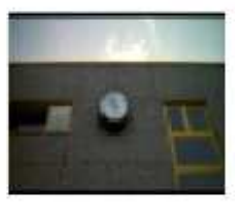

Clock3

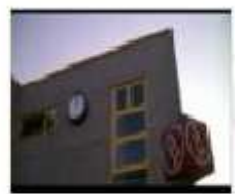

Clock6
Figure 5: Run-Lola-Run data set

Table 7: Overall Match Score (OMS) for "Run-Lola-Run" data set using Keyframe-based SIFT method

\begin{tabular}{llcc}
\hline $\begin{array}{l}\text { Representative } \\
\text { Image 1 }\end{array}$ & $\begin{array}{l}\text { Representative } \\
\text { Image 2 }\end{array}$ & OMS $_{1}$ & $P_{1}$ \\
\hline Clock1 & Clock2 & 10000 & 1.0000 \\
Clock1 & Clock3 & 170 & 0.9960 \\
Clock1 & Clock4 & 13 & 0.0213 \\
Clock1 & Clock5 & 0 & 0.0029 \\
Clock1 & Clock6 & 125 & 0.9918 \\
\hline Clock2 & Clock3 & 170 & 0.9960 \\
Clock2 & Clock4 & 13 & 0.0213 \\
Clock2 & Clock5 & 0 & 0.0029 \\
Clock2 & Clock6 & 125 & 0.9918 \\
\hline Clock3 & Clock4 & 0 & 0.0029 \\
Clock3 & Clock5 & 80 & 0.9766 \\
Clock3 & Clock6 & 97 & 0.9852 \\
\hline Clock4 & Clock5 & 117 & 0.9904 \\
Clock4 & Clock6 & 46 & 0.9105 \\
\hline Clock5 & Clock6 & 137 & 0.9934 \\
\hline & OMS $_{1}:$ Accuracy $=66 \%$ &
\end{tabular}

\subsection{Experiments on "Run-Lola-Run" data set}

We have also performed experiment to test the SIFT based method on the "Run-Lola-Run" dataset [9]. The representative images (of resolution $459 \times 366$ ) of this data set are shown in figure 5. We obtained an accuracy of $66 \%$ in the NID-image pairs (Refer to Table 7). We observed that the Clock4 image is falsely detected as Non-NID image with all images except the Clock5 and Clock6.

\section{Conclusions}

In this paper, we have evaluated two methods - Keyframebased method and Tomography-based method that uses a SIFT method, for the detection of Non-Identical Duplicate videos. Keyframe-based SIFT method provide significantly accurate results in the reasonable amount of computation time, while Tomography-based SIFT method does not perform well in the case of videos which has shaking artifacts. The future work will be to further investigate how Keyframe-based method and Tomography-based can be used to achieve better accuracy and efficiency on large size videos of different scales and of significantly different durations. It would also be interesting to explore the use of other methods such as wavelet-based method for detecting Non-Identical Duplicate video pair.

\section{References}

[1] A. Akutsu and Y. Tonomura. Video tomography : An efficient method for camera work extraction and motion analysis. In ACM International Conference on Multimedia, 1994.

[2] Pradeep K. Atrey, Yan Wei-Qi, and Mohan Kankanhalli. A scalable signature scheme for video authentication. In Journal of Multimedia Tools and Applications, 2006. To appear.

[3] Edward Chang, Chen Li, James Wang, Peter Mork, and Gio Wiederhold. Searching near-replicas of images via clustering. In SPIE Multimedia Storage and Archiving Systems IV, volume 3846, pages 281-292, 1999.

[4] Arun Hampapur and Ruud M. Bolle. Comparison of distance measures for video copy detection. In IEEE International Conference on Multimedia and Expo, 2001.

[5] Alejandro Jaimes, Shih-Fu Chang, and Alexander C. Loui. Duplicate detection in consumer photography and news video. In ACM International Conference on Multimedia, 2002 .

[6] Alejandro Jaimes, Shih-Fu Chang, and Alexander C. Loui. Detection of non-identical duplicate consumer photographs. In Pacific Rim International Conference on Multimedia, volume 1, pages 16-20, 2003.

[7] Yan Ke, R. Suthankar, and L. Huston. Efficient nearduplicate detection and sub-image retrieval. In $A C M$ International Conference on Multimedia, 2004.

[8] David G. Lowe. Distinctive image features from scale invariant key points. In International Journal of Computer Vision, 2004.

[9] J. Sivic and A. Zisserman. Video Google: A text retrieval approach to object matching in videos. In International Conference on Computer Vision, volume 2, pages 14701477, October 2003. 